\section{OP15 THE ASSOCIATION OF CROWDING WITHIN HOUSEHOLDS AND BEHAVIOURAL PROBLEMS IN CHILDREN; LONGITUDINAL DATA FROM THE SOUTHAMPTON WOMEN'S SURVEY}

\begin{abstract}
${ }^{1,2}$ Baird*, $^{1} \mathrm{R}$ Marsh, ${ }^{1} \mathrm{SR}$ Crozier, ${ }^{1,2} \mathrm{SM}$ Robinson, ${ }^{1,2} \mathrm{C}$ Cooper, ${ }^{1,2} \mathrm{KM}$ Godfrey, ${ }^{1,2} \mathrm{HM}$ Inskip. ${ }^{1} M R C$ Lifecourse Epidemiology Unit, University of Southampton, Southampton, UK; ${ }^{2}$ NIHR Southampton Biomedical Research Centre, University of Southampton and University Hospital Southampton NHS Foundation Trust, Southampton, UK
\end{abstract}

10.1136/jech-2018-SSMabstracts. 15

Background In England, nearly one child in ten lives in overcrowded housing. Crowding is likely to worsen with increasing population size, urbanisation, and the housing crisis. Children with behavioural difficulties are at increased risk of mental and physical health problems and poorer employment prospects later in life. We examined the relationship between crowding and behavioural problems in children aged 3 years within the Southampton Women's Survey.

Methods The SWS is a population-based cohort study of women aged 20-34 years assessed when not pregnant; those who became pregnant and their children $(n=3,158)$ were followed up. Mothers were interviewed when children were aged 2 years; they reported the number of people living in their home, the number of rooms and their perception of space within their home. A measure of crowding was derived as the number of people per room. At age 3 years, children's behaviour was assessed with the Strengths and Difficulties Questionnaire (SDQ). We used the total SDQ score as a continuous measure and univariate and multiple linear regression analyses were performed adjusting for confounding factors identified in a Directed Acyclic Graph: sex, single-parent family, maternal educational attainment, receipt of benefits, and occupation.

Results 2602 children (52\% boys) followed to age 3 years had complete data on crowding at age 2 years. The characteristics of the sample were broadly representative of the population in England. Median (IQR) SDQ score was 9 (6-12) and people-per-room was $0.75(0.6-1)$, with $327(13 \%)$ of mothers and children living in socially rented accommodation and $13.5 \%$ living in homes with $\geq 1$ people-per-room. Mothers' perception of space within their home tended to agree with the objective people-per-room measure of crowding. An increase of one household person-per-room was associated with a 1.30 increase SDQ points (95\% CI 0.70 to 1.90 ) adjusting for confounding factors. This relationship was partially mediated by greater maternal stress and depression, increased use of childcare, less sleep and strained parent-child interactions. Behavioural problems were greater in children who lived in socially-rented houses (by 2.69 SDQ points, $95 \% \mathrm{CI} 2.17$ to 3.20 ) than in children living in owner-occupied homes.

Conclusion Living in a more crowded home was associated with a greater risk of behavioural problems at age 3 years, independent of the effects of confounding factors. These findings support the need for improved housing for families, and suggest that families living in crowded circumstances might benefit from greater support.

\section{Mortality}

\section{OP16 DOES ETHNIC DIVERSITY CONFER PROTECTIVE EFFECTS ON POPULATION HEALTH? INTRA-UK COMPARISONS OF ETHNICITY AND MORTALITY}

${ }^{1} \mathrm{~L}$ Schofield, ${ }^{2} \mathrm{D}$ Walsh ${ }^{*},{ }^{3} \mathrm{Z}$ Feng, ${ }^{1} \mathrm{D}$ Buchanan, ${ }^{3} \mathrm{C}$ Dibbens, ${ }^{4} \mathrm{~J}$ Erdman, ${ }^{1} \mathrm{C}$ Fishbacher, ${ }^{5} \mathrm{G}$ McCartney, ${ }^{1} \mathrm{R}$ Munoz-Arroyo, ${ }^{2} \mathrm{~B}$ Whyte. ${ }^{1}$ Information Services Division, NHS National Services Scotland, Edinburgh, UK; ${ }^{2}$ Glasgow Centre for Population Health, Glasgow, UK; ${ }^{3}$ School of Geosciences, University of Edinburgh, Edinburgh, UK; ${ }^{4}$ NHS Greater Glasgow and Clyde, Glasgow, UK; ${ }^{5}$ HHS Health Scotland, Glasgow, UK

\subsection{6/jech-2018-SSMabstracts. 16}

Background The most likely underlying causes of the higher mortality in Scotland compared to England \& Wales (E\&W) and in Glasgow compared to Liverpool and Manchester have been identified recently as relating in large part to vulnerabilities created by adverse historical living conditions allied to detrimental political decision-making. However, it was also suggested that there is a protective effect in E\&W and Manchester of greater ethnic diversity, given the latter's association with lower-than-expected mortality among UK populations. We sought to assess the extent to which Scotland's 'excess' mortality (i.e. beyond that explained by deprivation) was attenuated by adjusting for ethnic diversity.

Methods The Scottish Longitudinal Study (SLS) and the ONS Longitudinal Study of E\&W were used. E-dataSHIELD methodology was applied to analyse the restricted access data. Risk of all-cause mortality (2001-2010) was compared between 3574 year-old residents of Scotland and E\&W, and between Glasgow and Manchester, using Poisson regression. Models adjusted for age, gender, socio-economic position (SEP) and an ethnicity* country of birth (CoB) interaction. $\mathrm{CoB}$ has been shown to be important in explaining differences in ethnic mortality related to the 'healthy migrant effect'.

Results $18 \%$ of the Manchester sample was classed as non-White compared to only $3 \%$ in Glasgow. The equivalent figures for E\&W and Scotland were $10.4 \%$ and $1.2 \%$ respectively. The mortality Incident Rate Ratio (IRR) was 1.33 (95\% CIs 1.13, 1.56) in Glasgow compared to Manchester. This reduced to 1.25 (95\% CI 1.07 to 1.47) after adjustment for SEP, and further reduced to 1.20 (95\% CI 1.02 to 1.42 ) after adjustment for ethnicity*CoB. The equivalent figures for Scotland compared to E\&W were: 1.18 (95\% CIs 1.16 to 1.21 ) overall, reduced to 1.08 (95\% CIs 1.05 to 1.10) after adjustment for SEP, and then to 1.04 (95\% CIs 1.02 to 1.07) after adjustment for ethnicity* $\mathrm{CoB}$. Across all samples, NonWhites born outside UK/ROI had a lower risk of mortality than Whites born in UK/ROI; however, among the Scottish samples only, non-Whites born in UK/ROI had a higher risk of mortality compared with Whites born in UK/ROI - IRR 1.77 (95\% CIs 1.10 to 2.85 ) for Scotland, 3.10 (95\% CIs 1.28 to 7.51 ) for Glasgow.

Conclusion The research supports the hypothesis that greater diversity in ethnicity and migration status plays a partial role in explaining Scottish excess mortality. In Glasgow's case, however, a large excess remains: thus previously articulated policy implications such as addressing poverty, vulnerability and inequality still apply. Further research is warranted into the relatively high mortality of non-Whites born in UK/ROI and resident in Scotland. 